\title{
Development of a model for laparoscopic cholecystectomy video assisted training. A randomized study ${ }^{1}$
}

Francesco Mongelli', Davide La Regina", Michele Marengo"', Ramon Pini' ${ }^{\prime \prime}$, Andrea Saporitov, Maurice FitzGerald ${ }^{\mathrm{vI}}$, Matteo Di Giuseppe ${ }^{\mathrm{VII}}$

'MD, Department of Surgery, Ospedale Regionale di Bellinzona e Valli, Switzerland. Manuscript writing. "MD, Department of Surgery, Ospedale Regionale di Bellinzona e Valli, Switzerland. Critical revision.

"'MD, Department of Surgery, Ospedale Regionale di Bellinzona e Valli, Switzerland. Acquisition of data.

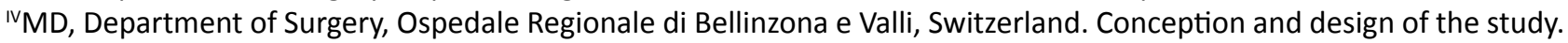
${ }^{\vee} M D$, Division of Anesthesiology, Ospedale Regionale di Bellinzona e Valli, Switzerland. Statistical analysis.

V'MD, Department of Surgery, Ospedale Regionale di Bellinzona e Valli, Switzerland. Manuscript preparation.

VIIMD, Department of Surgery, Ospedale Regionale di Bellinzona e Valli, Switzerland. Manuscript preparation. Final approval.

\section{Abstract}

Purpose: To identify the surgical trainee benefits through the use of video assisted training (VAT).

Methods: Twelve cases of uncomplicated laparoscopic cholecystectomy (LC) were selected. Edited video segments focused on essential intra-operative anatomical structure identification and critical surgical steps. Evaluation of these videos was constructed on a scoring system based on pre and post-teach tests.

Results: The mean value of the pre-teach score was $33.2 \pm 18.0$ points and in the post-teach test the mean value was $66.7 \pm 9.7$ points. A statistically significant difference was seen when comparing pre and post-teach results $(p<.00001)$.

Conclusion: The widespread and the systematic use of video assisted learning may be a useful and economic tool in adjunct to the surgical training techniques practiced until now throughout the surgical community.

Key words: Cholecystectomy, Laparoscopic. Training. 


\section{- Introduction}

One of the most difficult challenges in surgical resident training is the transition from surgical theory to surgical skills in the operating room. latrogenic errors and surgical complications are directly associated and proportional to the inexperience of the surgeon ${ }^{1}$. Surgical training is often hampered by the technical difficulty of a case, case quantity and the operating room resources of the institute providing such a service ${ }^{2}$. The willingness of such institutes to initiate a surgical training experience that mirrors the real life intervention, is of obvious benefit, not to just the surgical resident but also for patients and the system as a whole as it would streamline training, reduce intra-operative errors and be more cost effective ${ }^{1 .}$

Although widespread video assisted training (VAT) still does not feature in standard surgical training to a qualifiable extent, with the classic methods of intra-operative "trial and error" and "see one, teach one, do one" still dominating the surgical training experience ${ }^{3,4}$. Our study aims at identifying the surgical trainee benefits through the use of video assisted training, with the hope of identifying an effective and relevant trainee aid that is resource cheap and educationally effective.

\section{- Methods}

We used a within-subjects randomized experimental design involving 14 surgical residents with maximum 2 years of surgical experience. The candidates were selected and subsequently tested at different times between January and April 2018 at San Giovanni Hospital, Bellinzona, Switzerland.

The training videos were comprised of 12 cases selected at random out of a database of 25 recorded laparoscopic cholecystectomy (LC). All of the cases were uncomplicated elective procedures performed by the same senior surgeon and a senior resident not included in the study.

Evaluation of these videos was based on two tests: the pre-teach exam was administered prior to a candidate viewing the video. It required the subject to answer questions based on diagrams and photographs of various anatomical structures and technically relevant areas necessary to identify for completion of a LC (ie. correct placement of trocars, triangle of callot, relevant anatomy, clips placement and line of dissection) (Figure 1).

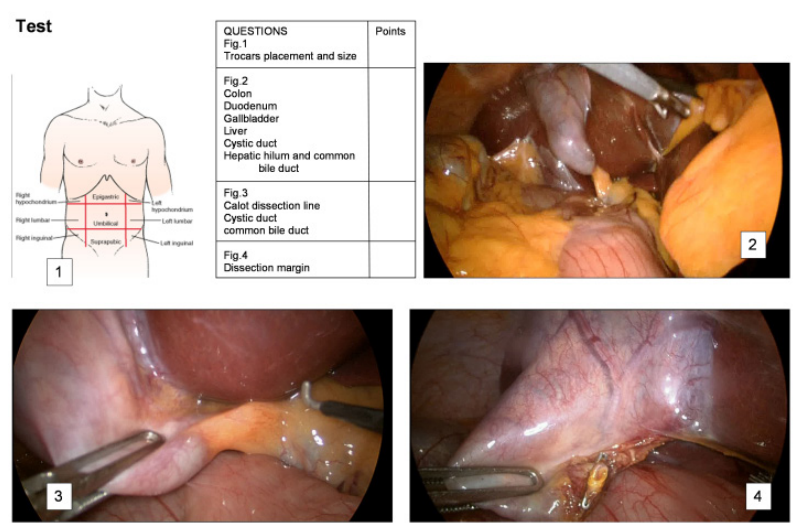

Figure 1 - The test given to residents, divided in 4 sections. In the first it was necessary to decide the trocars placement and sizes. In the sections 2, 3 and 4 it was necessary to identify several anatomical structures. The given score was then calculated and filled in the middle column.

Following completion of the pre-teach questionnaire, the senior surgeon narrated the video segments with each video tailored and edited to focus on four essential intra-operative segments, each segment concentrating on anatomical structure identification and critical surgical techniques, with all segments being in chronological order. The subjects were then assigned 20 minutes to study the LC training video. The second or post-teach test was administered once the candidate had studied 
the laparoscopic video tutorial with similar photo based exam questions related to the cholecystectomy. The pre and post-test were randomly assigned from 12 possible tests, each being generated to be equatable in content matter and difficulty. The use of a questionnaire with the laparoscopic photographs based upon the video of the laparoscopic procedure just watched by the candidate, was avoided in order to exclude bias and to achieve a standardised, consistent level of difficulty. The exams involved 4 different randomly assigned LC, each composed by 4 exercises. A scoring system from 1 to 5 for every exercise was assigned.

\section{Randomization and statistical analysis}

Randomization and statistical analyses were performed using MedCalc for Windows, version 15.0 (MedCalc Software bvba, Ostend, Belgium; https://www.medcalc.org; 2016). The randomization was performed assigning the questionnaire according to the value given by the software. The student t-test was used to compare means across variables. The statistical significant difference was fixed at $p=.01$. The standard deviation and other descriptive values were also calculated. The method used to graphically depict the median, upper and lower quartile, upper and lower extreme between groups was the box-plot.

\section{Results}

The attendance of the participants was $100 \%$. The mean value of the pre-teach score was $33.2 \pm 18.0$ points and in the post-teach test the mean value was $66.7 \pm 9.7$ points. Details of the surgical residences' scores are shown in Table 1. A statistically significant difference was seen when comparing pre and post-teach results $(p<.00001)$. Median, upper and lower quartile, upper and lower extreme are reported in the Figure 2.
Table 1 - Scores before and after the video training are listed. The $p$-value indicate the statistically significant difference found between groups.

\begin{tabular}{llll} 
Residents & $\begin{array}{l}\text { Pre-teach } \\
\text { score }\end{array}$ & $\begin{array}{l}\text { Post-teach } \\
\text { score }\end{array}$ & P value \\
\hline N01 & 46 & 69 \\
N02 & 36 & 58 \\
N03 & 55 & 80 \\
N04 & 47 & 71 \\
N05 & 30 & 60 \\
N06 & 49 & 80 \\
N07 & 16 & 60 \\
N08 & 14 & 67 \\
N09 & 18 & 56 \\
N10 & 25 & 71 \\
N11 & 66 & 80 \\
N12 & 9 & 53 \\
N13 & 41 & 74 \\
N14 & 13 & 55 \\
Mean Value & $33.2 \pm 18.0$ & $66.7 \pm 9.7$ \\
$( \pm S D)$ & & & $<.000001$ \\
\hline SD & & & \\
\hline
\end{tabular}

SD = Standard Deviation

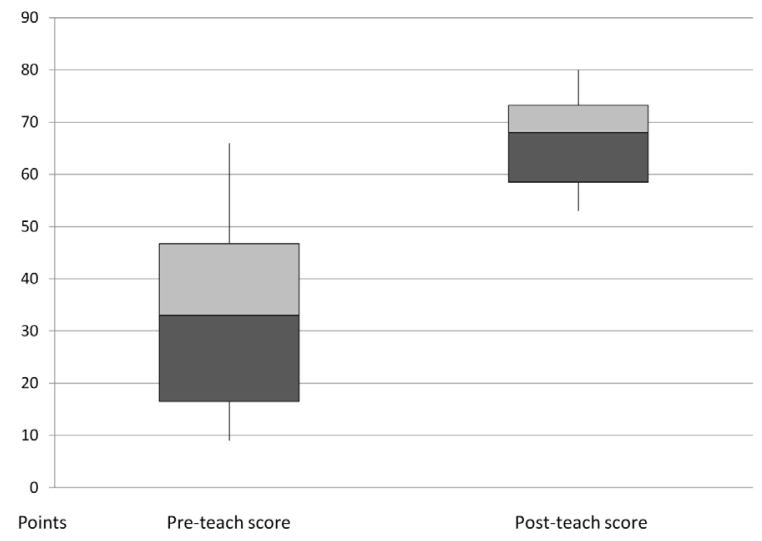

Figure 2 - The box plot describe the distribution of results obtained in the pre and post-teach tests by the surgical residents. 


\section{Discussion}

The last two decades have seen a dynamic change in the surgical therapy of cholecystectomy. Although the advent of the laparoscopic technique has brought great advantages for both patient and surgeon, advances and use of this technique to educate trainee surgeons still has not been optimised in a ubiquitous fashion.

Numerous studies have identified experience related error and difficulty observed by trainee surgeons, most notably at the initial stages of training 5 . The "learning curve» for surgical trainees is a known entity as trainees familiarise themselves with all aspects of the operation with previous studies having demonstrated a clear proportionality between practice and patient outcome ${ }^{1}$. VAT of LC, demonstrated in an informative and educational manner, to offer great advantages, as they are reproducible, inexhaustible and may be displayed and explained to trainee surgeons, without the use of hospital resources or patient risk ${ }^{6}$.

The results from our study support our aim in identifying the beneficial use of video tutorials in the education of surgical trainees. The fact that pre-teach to post-teach results showed a positive improvement in all test subjects is promising and is a clear indicator of the usefulness of VAT in LC training. We confirm the evidence offered by prior studies in that it gives a clear educational benefit in the acquisition of cognitive skills, necessary for motor and laparoscopic skills and highlights the potential role of VAT as an introductory and preparative precursor to operatory room learning ${ }^{7}$. Extrapolating our test results and applying them in a more formal manner to the overall training of surgical residents may be a time and cost effective tool to augment intraoperative teachings.

A surgical trainee may require up to an hour to observe a full laparoscopic cholecystectomy, with the crucial moments spread out throughout the operation. With the use of VAT, the same resident may observe multiple interventions within the same time allotment, focusing on particular surgical techniques to recognise and subsequently apply. VAT also permits the documentation and subsequent dissemination of case specific details; complications, anatomical or pathological rarities, particular laparoscopic techniques to be aware of, or errors to avoid. The simplicity of such LC tutorials would allow surgical education in an efficient and targeted way, allowing senior surgeons to teach and demonstrate case specific details, that could take months or years for a surgical resident to encounter by following operatory room caseby-case training.

The weaknesses of our study are based majoritively on our small sample size and subsequent demographic variation. We examined a relatively small population number with a potentially variable degree of preceding laparoscopic experience. However this does not account for the fact that there was a statistically significant improvement across the board.

Apart from the advantage to both the surgical trainee and the cost benefits for the training institution, it should be noted the added gain for patients. It goes without saying that any surgical intervention, subject to human error, varying degrees of experience and competency, may benefit from a systemic approach to improving pre-operative surgical knowledge. The use of VAT to develop the acquisition of laparoscopic skills, could reduce the amount of human error associated with the learning curve, thereby contributing positively to patient outcomes. Additionally the use of a safe, inexpensive training technique, that does not involve patient risk would be of tremendous benefit to the surgical community. 


\section{- Conclusion}

The widespread and the systematic use of video assisted learning may be a useful and economic tool in adjunct to the surgical training techniques practiced until now throughout the surgical community.

\section{- References}

1. Cecilio-Fernandes $D$, Cnossen $F$, Jaarsma DADC, Tio RA. Avoiding surgical skill decay: a systematic review on the spacing of training sessions. J Surg Educ. 2018 Mar-Apr;75(2):471-80. doi: 10.1016/j. jsurg.2017.08.002.

2. Voitk AJ, Tsao SG, Ignatius S. The tail of the learning curve for laparoscopic cholecystectomy. Am J Surg. 2001;182(3):2503. PMID: 11587686.

3. Ferrarese A, Gentile V, Bindi M, Rivelli M, Cumbo J, Solej M, Enrico S, Martino V. The learning curve of laparoscopic cholecystectomy in general surgery resident training: old age of the patient may be a risk factor? Open Med. 2016;11(1):489-96. PMID: 28352841.

4. Mota P, Carvalho N, Carvalho-Dias E, João Costa M, Correia-Pinto J, Lima E. Videobased surgical learning: improving trainee education and preparation for surgery. J Surg Educ. 2018 May-Jun;75(3):828-35. doi: 10.1016/j.jsurg.2017.09.027.

5. Gawande AA, Zinner MJ, Studdert DM, Brennan TA. Analysis of errors reported by surgeons at three teaching hospitals. Surgery. 2006;133(6):614-21. PMID: 12796727.

6. Singh $\mathrm{P}$, Aggarwal $\mathrm{R}$, Tahir $\mathrm{M}$, Pucher $\mathrm{PH}$, Darzi A. A randomized controlled study to evaluate the role of video-based coaching in training laparoscopic skills. Ann Surg. 2015;261(5):862-9. doi: 10.1097/ SLA.0000000000000857.

7. Wulf G, Shea C, Lewthwaite R. Motor skill learning and performance: a review of influential factors. Med Educ. 2010;44(1):7584. doi: 10.1111/j.1365-2923.2009.03421.x.

\section{Correspondence:}

Francesco Mongelli

Department of Surgery, San Giovanni Hospital via Ospedale, Bellinzona

6500 Switzerland

Phone: +41918119465

francesco.mongelli@mail.com

Received: Feb 18, 2018

Review: Apr 15, 2018

Accepted: May 14, 2018
Conflict of interest: none

Financial source: none
${ }^{1}$ Research performed at Department of Surgery, San Giovanni Hospital, Bellinzona, Switzerland. 